\title{
Imaginarios sociales sobre las violencias de género de los habitantes de una comunidad rural del departamento del Huila (Colombia)
}

Social Imaginaries about Cender-Based Violence of Inhabitants of a Rural Community in the State of Huila, Colombia.

\section{Por: Juan Felipe Cuzmán, ${ }^{1}$ María Alejandra Campos Caicedo, ${ }^{2} \&$ Maudy Soledad Ortega ${ }^{3}$}

1. Psicólogo, Especialista en Cestión de Procesos Psicosociales, Magister en Administración y Planificación Educativa, Docente Universitario y Psicoterapeuta. Pitalito Huila Colombia. Orcid: https://orcid.org/0000-0002-2161-6344 Scholar: https://scholar.google.es/citations?hl=es\&user=tklgTPgAAAA] Contacto: efguzmanp@gmail.com

2. Psicóloga, Corporación Universitaria Minuto de Dios. Orcid: https://orcid.org/0000-0002-6185-2392 Scholar; https://scholar.google.com/ citations?hl=es\&user=ONb8ns|AAAA] Contacto: mariaaleja2992@gmail.com

3. Psicóloga, Corporación Universitaria Minuto de Dios. Orcid: https://orcid.org/0000-0001-7018-4739 Scholar; https://scholar.google.com/citations?hl=es\&user=R KCZfAAAAA] Contacto: maudy2195@gmail.com

GOPEN ACCESS

\section{(c) (1) $\Theta(9$}

Copyright: (C) 2020 El Ágora USB.

La Revista El Ágora USB proporciona acceso abierto a todos sus contenidos bajo los términos de la licencia creative commons Atribución-NoComercial-SinDerivar 4.0 Internacional (CC BY-NC-ND 4.0)

Tipo de artículo: Investigación

Recibido: marzo de 2020

Revisado: abril de 2020

Aceptado: junio de 2020

Doi: $10.21500 / 16578031.5133$

Citar así: Guzmán, J., Campos Caicedo, M. \& Ortega, M. (2020). Imaginarios sociales sobre las violencias de género de los habitantes de una comunidad rural del departamento del Huila (Colombia). El Ágora USB, 20(2). 102-117

Doi: $10.21500 / 16578031.5133$

\section{Resumen}

El uso de lenguajes sexistas y discriminatorios hacia la mujer, sobre todo en los roles desempeñados por el hombre y la mujer en el hogar, hacen parte de los imaginarios que en la comunidad de la Vereda Belén del municipio de Isnos (Huila) se evidencian sobre las violencias de género; además, se observó la emergencia de ciertos imaginarios radicales construidos desde la familia, a partir de experiencias y vivencias personales con sus padres como modelos de crianza.

Palabras clave: imaginarios sociales; violencia; género; familia

\section{Abstract:}

The use of sexist language, which is discriminatory towards women, especially in the roles played by men and women, at home, are part of the imaginaries that in the community of Belén Village, in the Municipality Isnos, Huila, about gender-based violence is made evident. Besides, the emergence of certain radical imaginaries built from the family, based on personal experiences, and experiences with their parents, as parenting models, was observed.

Keywords: Social Imaginaries; Violence; Gender; and Family. 


\section{Introducción}

La violencia de género representa una problemática social que trasciende las esferas económicas en Colombia, al tiempo que se convierte en un padecimiento generacional que marca un hito en la historia del país, con énfasis en los relatos de las personas que se enfrentaron al conflicto y aprendieron a desarrollar acciones o conductas desde la apología al machismo y al control en la familias.

Esta situación dentro de la interacción social, produce en los miembros esquemas mentales cotidianos de maltrato, misoginia y actuaciones naturalizadas aprendidas, lo cual se convierte en imaginarios sociales que trascienden el entendimiento y la lógica. Al respecto Castoriadis, citado por Riffo Pavón (2016, p.65), expresa que "lo imaginario no es imagen de. Es creación incesante y esencialmente indeterminada de figuras/formas/imágenes". De esta forma se percibe que los imaginarios son construidos socialmente a partir de percepciones personales equivocadas para unos pero veraces para otros.

En el caso de la violencia de género, los imaginarios se construyen en la psique de la familia a través del tiempo y son reforzados bajo ideas prefabricadas por los individuos y erróneamente aceptadas por el colectivo social que son replicadas a su tiempo y generan impactos negativos en la vida de las víctimas afectadas por esta problemática.

La violencia de género es multidimensional y se incorpora a todos los ámbitos sociales y los contextos culturales en los que se presenta, comprendiéndola como un entramada de fenómenos dinámicos que son producidos por la interacción con sus relaciones interpersonales y afectivas, lo cual forja en su accionar una especie de dependencia emocional que por temor o por monotonía se acepta para llenar los vacíos que derivan de estas relaciones. En este sentido, las historias de vida de las mujeres y los hombres maltratados o víctimas de la violencia dependen mucho de la forma en la cual se desarrollan sus relaciones más inmediatas, es decir, la manera como las relaciones de género se dan en la cotidianidad de quién maltrata o agrede. Consecuentemente, su interacción depende de factores que intervienen activamente en la causa y en potenciadores que son las que impulsan la acción violenta o en el riesgo de materializarla.

En Colombia la familia se representa por una parte por un perfil tradicional caracterizado por un conjunto de estructuras sociales con múltiples estilos y por ende en constante evolución y por otra parte con una gran cantidad de expresiones a nivel afectivo, pasional, de poder, de intereses y de diferencias. En este sentido en Colombia la familia es una sociedad donde se desarrollan una serie de conflictos que están unidos a un sin número de situaciones inconclusas que terminan en imaginarios los cuales se convierten en segregadores y discriminatorios, como lo menciona Cornelius Castoriadis (citado por Cruz, Ballén, Medina \& Rodríguez, 2014), cuando expresa que las construcciones instituidas en la psique a través de un largo proceso de la fabricación del individuo social le dan la oportunidad desde su primer hábitat natural de dar sentido al mundo, lo cual implica crear una representación social del mismo y del mundo para sí mismo.

\section{Situación problemática}

Para la Organización Mundial de la Salud [OMS] (2017), la violencia de género se define como:

El uso deliberado de la fuerza física o el poder, ya sea en grado de amenaza o efectivo, contra uno mismo, otra persona o un grupo o comunidad, que cause o tenga muchas probabilidades de causar lesiones, muerte, daños psicológicos, trastornos del desarrollo o privaciones (p.1).

La violencia de género es considerada multidimensional y afecta todas las esferas sociales, lo que hace que se convierta en una afectación a la vida de la víctima, pero también del entorno que la rodea. 
En el contexto internacional, investigaciones como las realizadas por la OMS (2018), las mujeres son las mayores víctimas de maltratos y denigraciones en el mundo, la mayoría de los casos ha padecido violencia sexual, mutilaciones en alguna parte de su cuerpo, en especial en órganos sexuales, denigraciones, trata de blancas, y la mayoría de ellas han sido víctimas desde niñas, padeciendo hasta a muerte; según los resultados del informe, se tiene que, las mujeres son las mas afectadas en situaciones de trata de mujeres en un 51\%, en el mundo, cómo también se tiene que el $71 \%$ de las mujeres víctimas son niñas y son agredidas sexualmente.

Por otro lado, en los resultados del informe, la Organización de las Naciones Unidas [ONU] (2019), menciona que

El 35 por ciento de las mujeres de todo el mundo ha sufrido violencia física y/o sexual por parte de un compañero sentimental o violencia sexual por parte de otra persona distinta a su compañero sentimental, (estas cifras no incluyen el acoso sexual) (p.14).

Según esto, se deduce que la mayoría de los casos son resultados de problemáticas como violencia intrafamiliar, maltratos escolares y sociales. Los datos son preocupantes, dado que los esfuerzos por comprender este fenómeno han sido significativos en todo el mundo, dentro del estudio, la ONU menciona que en la mayoría de los casos, las mujeres que son agredidas padecen altas tasas de depresión, y mayor riesgo de autolesiones o de practicarse abortos o de contraer enfermedades de trasmisión sexual o de contagio de VIH.

De igual manera, según los resultados obtenidos por la ONU (2019), países como Oriente Medio y Africa del Norte, demuestran que,

Los hombres durante su infancia presenciaron un comportamiento violento de su padre hacia su madre o sufrieron alguna forma de violencia doméstica, y presentan una probabilidad notablemente superior de actuar de forma violenta como compañeros sentimentales en las relaciones mantenidas en su vida adulta (p.21).

En este caso, el aprendizaje por modelamiento de conductas genera la réplica de actos violentos a nivel fisico, psicológico y emocional; lo mismo que los resultados obtenidos por el informe de la ONU mencionan que cerca de 650 millones de mujeres y niñas se casaron antes de cumplir los 18 años, mas de cuatro de cada diez mujeres jóvenes fueron obligadas a contraer matromonio con personas adultas, o fueron negociadas por algun tributo económico, ocasionando problemáticas como embarazos precoces y al aislamiento social, obstaculizando la escolarización, limitando las oportunidades de las niñas y aumentando el riesgo de sufrir violencia doméstica.

Como se observa, las estadísticas a nivel mundial remarcan la enorme necesidad de establecer acciones desde el estado para atender esta problemática de carácter social considerada delito en algunos países del mundo; según la ONU (2013), "la violencia física o sexual es un problema de salud pública que afecta a más de un tercio de las mujeres en el mundo" por lo que las instituciones a cargo de la protección y defensa de la mujer están atentos a la minimización de los riesgos de violencia de género mediante acciones jurídicas concretas contra los agresores quienes en la mayoría de las ocasiones son liberados por la permisividad de las leyes, a pesar de que las experiencias predicen que estos actos violentos desencadenan en finales trágicos para la vida de las víctimas,

Se estima que de las 87,000 mujeres que fueron asesinadas globalmente en el 2017, en más de la mitad (50,000-58\%) los culpables fueron sus parejas o miembros de la familia de las víctimas. Lo que quiere decir que 137 mujeres alrededor del mundo son asesinadas a diario por un miembro de su familia. Más de un tercio $(30,000)$ de las mujeres asesinadas en el 2017 fueron exterminadas por su actual o ex pareja, (ONU, 2019). 
Igualmente, en estos datos de la ONU (2019), que no solo en el contexto familiar se padece la violencia de género, los maltratos psicológicos de muchas niñas en su contexto educativo demuestran la poca relevancia a nivel mundial que se le brinda al acoso escolar. Además, en ese mismo informe se presenta que en el mundo, uno de cada tres estudiantes (de 11 años y de entre 13 y 15 años de edad), sufrió el acoso de compañeras o compañeros en el centro educativo al menos un día, lo cual indica que los niños y las niñas tienen igual riesgo de sufrir acoso escolar. Se evidencia, según lo descrito en las investigaciones citadas, que los hombres son más víctimas de acoso físico y las mujeres son más víctimas de acoso psicológico y privación emocional. En este sentido, se describe que las niñas toleran rumores desagradables por parte de sus compañeros sobre su aspecto físico, burlas acerca de su forma de pensar o sentir, lo que limita su libre expresión y ocasiona pérdidas y daños irreparables en su autoestima y autoimagen con influencia negativa en la construcción de su proyecto de vida.

Esta situación genera una limitante en la mujer en su derecho a la educación, y en múltiples ocasiones la pérdida de la vida por decisión propia. De igual manera en los estudios se demuestra que más o menos un $10 \%$ de la población femenina ha sufrido ciberacoso con acoso sexual a través de las redes sociales. En cuanto a la violencia de género, los países latinoamericanos se han caracterizado por tener los más altos niveles de maltrato hacia la mujer y casos de feminicidios. Al respecto Fernández Moreno (2017), expone que durante el año 2014 Hondura fue el país de la región con el mayor número de feminicidios (531 en 2014), lo cual representa 13,3 feminicidios por cada 100.000 mujeres, lo sigue Republica Dominicana, con 200 feminicidios. Igualmente, expresa este autor que para el año 2015, se observó que en países como Argentina asesinaron a una mujer cada 30 horas, los cuales sumaron para el año 2016, 275 mujeres muertas.

Además, continúa el autor antes citado que para el año 2016, países como Bolivia registró 48 asesinatos de mujeres por violencia machista, el Salvador 407 feminicidios, lo mismo que en Brasil se presentó un promedio de 15 mujeres asesinadas cada día, por el simple hecho de ser mujer. En Chile se registraron 30 casos de feminicidios; en Costa Rica 14 homicidios de mujeres de 15 años de edad y más, asesinadas por razones de género; al mismo tiempo, en Ecuador se observaron 97 feminicidios.

En Colombia la situación es similar y el fenómeno de la violencia de género no es una problemática reciente, su estudio y los esfuerzos para visibilizarla se sustentan en la historia violenta de Colombia, sin embargo, ha sido vista desde diferentes contextos y posiciones como un problema de carácter social al cual no se le ha dado la suficiente importancia y por ende ha sido naturalizado por las comunidades como parte de un arraigo cultural trasmitido inter generacionalmente y con múltiples causas centradas en factores psicosociales. En este país los estudios realizados por el Ministerio de Protección Social (2018, p.21), demuestran que, en el Departamentos como San Andrés y Vaupés, se presentó un incremento en el reporte de casos de violencia, siendo 247 casos, de los cuales 218 de las agresiones resultan de ciudadanos procedentes de Venezuela. Por otra parte, el $30 \%$ corresponden a casos de violencia física, el $53 \%$ a negligencia y abandono y el $17 \%$ a violencia sexual.

En estudios realizados por Sistema Nacional de Vigilancia en Salud Pública [SIVIGILA], (2018, p.21), se consiguio que el 58,5\% de los casos sospechosos de violencias de género e intrafamiliar se distribuyeron en siete entidades territoriales de la siguiente forma: Antioquia con 5.332 casos (13,9\%), Valle del Cauca 4.879 casos (12,7\%), Bogotá 3.632 casos (9,5\%), Cundinamarca 2.986 casos (7,8 \%) Santander 2.099 casos (5,5\%), Huila 1.847 casos (4,8 \%) y Nariño 1.600 casos (4,2 \%)", en esta región los casos reportados tienen un nivel alto de incidencia por violencia de género a nivel intrafamiliar.

De esta forma, según el Instituto Nacional de Salud (2018), los casos de violencia de género en Colombia ascienden a 76,8 por 100.000 habitantes, de los cuales el informe señala que los departamentos con mayor incidencia son Vaupés, Amazonas y Casanare con 180, 177 y 169 casos por 100.000 
habitantes respectivamente. En el departamento del Huila, investigaciones realizadas por Secretaría de Salud Departamental (2018), demuestran en torno a la temática de la violencia de género, 11 casos mortales asociadas a Violencias de Cénero, ocho de ellos por sospecha de Negligencia/Abandono y tres asociadas a violencia física, dos en menores de edad y otro rotulado como feminicidio. De igual manera expresa el informe que hubo notificación de 5.327 casos, los cuales fueron confirmados por las entidades de salud, asociados a conductas de abuso, violencia y maltrato. Estas cifras reflejan que existe una tasa de incidencia de 2.844 casos de violencia de género distribuidos en el norte del departamento del Huila, en los municipios de Neiva, Palermo, Algeciras, Gigante, Santa María, Aipe, Iquira, Teruel, Agrado, Tesalia, Baraya, Villavieja, Yaguará, Tello, Rivera, Hobo, Campoalegre, Santa María, Paicol, Nátaga, Colombia;

Por otro lado, el informe de 2018 presenta que para el sur del Departamento de Huila, se presentaron en la misma vigencia 1.788 casos reportados de violencia de género, distribuidos entre los municipios de Garzón, Plata, Paicol, Nátaga, Isnos, Acevedo, Suaza, Guadalupe, La Argentina, Timana, Tarqui, Saladoblanco, Palestina, Pital, Oporapa, Altamira, Elías. Respecto al municipio de Isnos, estudios como el realizado por la Secretaría de Salud Departamental por medio del SIVICILA (2018), expresan que se reportan 114 casos de violencia intrafamiliar, además de que el plan de desarrollo municipal para el año 2012-2015, demuestran que "del total de la población para el 70,4\% de las personas no cuentan con información sobre atención sobre Violencia Intrafamiliar y violencia sexual" (p.90), al igual que el plan de desarrollo municipal para el periodo actual 2016-2019, demuestran que el $67.1 \%$ de las mujeres son vulneradas y el $32.9 \%$ de los hombres son agresores. Igualmente, en este informe se evidencia que para el año 2018, se reportaron 17 casos de abuso sexual en el municipio, además de que, se presentaron 661 casos de vulneración de derechos de los N/N/A, de los cuales 80 pertenecen al género femenino y 84 son de género masculino, con causas como desplazamiento forzado, homicidio, amenaza y otros hechos victimizantes,

Para el contexto donde se desarrolla la presente investigación, reconocida como la vereda Belén del municipio de Isnos, se reportaron a la Comisaria de Familia para el año 2018, veintiún (21) casos de violencia de género, que se presentan por diversas causas, y diferentes motivos la denuncia; al tiempo, se tiene que en la vereda existe una abstención por la denuncia, lo que incrementa la problemática, pues no se puede tener una cifra concreta de los casos de violencia de género que están siendo enmascarados por las víctimas. Es por esto, que, a raíz de la problemática evidenciada en lo referente a la violencia de género, se pretende ahondar en los imaginarios que tiene la población acerca del tema y se busca desarrollar la presente investigación, para dar respuesta a la siguiente pregunta de investigación:

¿Cuáles son los imaginarios sociales sobre las violencias de género de los habitantes de la vereda Belén del municipio de Isnos (Huila)?

\section{Fundamentos teóricos}

Reconocer los imaginarios sociales de la violencia de género en Colombia es realizar un recuento sociocultural en todo el marco histórico de las regiones, teniendo en cuenta las representaciones tangibles e intangibles que ha dejado la violencia en el país; además de tener en cuenta las percepciones sociales que afectan los imaginarios colectivos que se derivan de la problemática, en torno a esto, las huellas de violencia en tanto mujeres como hombres, requiere ser evaluadas desde las dimensiones o categorías de análisis propias de las ciencias sociales y humanas.

De la problemática se reconoce que la palabra violencia según la Real Academia de la Lengua Española, (Águila Gutiérrez, Hernández Reyes y Hernández Castro, 2016), se origina del latín "vis" cuyo significado es "fuerza", frente a esto, la violencia quiere decir "fuerza intensa contra algo", los factores que derivan de la problemática están evidenciados a través de medios que permean la aparición de las conductas negativas en el medio donde se desarrolla el individuo, siendo de orden 
social como los conflictos, pérdida de valores espirituales, morales y éticos, sobrepoblación, desplazamientos forzosos, problemáticas de desigualdades, estímulos violentos a través de los medios de comunicación masivos, los cuales son modelos representativos que tienden a imitarse.

En torno a esto, la OMS (2017), reconoce como violencia de género

Todo acto de violencia basado en el género que tiene como resultado posible o real un daño físico, sexual o psicológico, incluidas las amenazas, la coerción o la privación arbitraria de la libertad, ya sea que ocurra en la vida pública o en la vida privada.

Consecuentemente, la violencia se enmarca en una construcción socialmente aprendida a través de las bases familiares y sociales donde interactúa el individuo y a partir de esto, replica el fenómeno desde su propia manifestación conductual, por medio de las expresiones físicas y simbólicas de una violencia que adquieren materialidad concreta e imaginaria que afecta las trasformaciones sociales frente a las realidades diferentes según las construcciones permanentes de sentido.

Por lo tanto, los imaginarios sociales de violencia de género no solo son representaciones que se enmarcan en la acumulación histórica que definen el papel trasformador de la cultura en la sociedad, sino que derivan de su identidad enmarcada bajo los parámetros sociales de los valores personales, los cuales son analizados mediante la afectación social que tiene la violencia en la sociedad y en especial en las familias, donde se definen las conductas violentas de los individuos desde lógicas que le dan forma a los imaginarios colectivos en la problemática que se incluye como social.

De este modo, se genera en el contexto de las familias afectaciones muchas veces enmascaradas y retenidas bajo presiones, temores y concepciones de dominancia en los géneros desde el masculino sobre el femenino en la mayoría de los casos, siendo más impactante sus consecuencias.

Al respecto Águila Gutiérrez, Hernández Reyes y Hernández Castro (2016) exponen:

La violencia de género puede darse en varios escenarios, tales como el laboral, escolar, espacios comunitarios y de convivencia entre vecinos, de las relaciones familiares y de pareja, resultando más frecuente su en estos dos últimos. Puede manifestarse de diversas formas, lo cual da lugar a los distintos tipos de violencia, entre los cuales destacan la violencia física, psicológica (forma más sutil de violencia), sexual, económica, entre otras (p. 702).

Por otro lado, autores como Segato (2003) mencionan que la violencia de género obedece a unas estructuras de orden muy arcaico en sus origenes y que no evolucionan ante las exigencias de los cambios sociales, sino que responden de manera lenta, lo cual afecta de manera significativa la convivencia y la armonía dentro de los hogares. Esto hace que la violencia sea una afectación indirecta que conlleva a una desestructuración de las relaciones personales y en algunos casos a comportamientos aprendidos de indefensión por parte de las víctimas.

Por lo tanto, frente a la concepción que tiene la violencia de género en una comunidad, se reconoce el entramado del poder acumulado en sus acciones, lo cual hace que se construyan imaginarios sociales de normalización de la problemática, que conllevan a enmascarar o a disimular su presencia, es decir se genera una incapacidad de identificar la violencia e intervenirla.

Debe señalarse, que la violencia de género se considera un proceso aprendido por el agresor en el seno familiar. Por una parte lo aprende debido a vacíos afectivos y de abandono parental, en el cual el agresor por haber sido abandonado por su progenitor desencadena una personalidad maltratadora y por otra parte, por una niñez llena de maltratos y violencia que genera en el adulto daños emocionales que influyen directamente con su interacción como pareja. Esta situación se convierte en un círculo recursivo, por cuanto una familia conformada por maltratados dará como resultado una familia con ambientes de violencia y maltrato. 


\section{Al respecto Alencar-Rodrigues y Cantera (2012), expresan que:}

Existen algunas características individuales que constituyen un factor de riesgo para que las personas ejerzan la violencia contra la pareja. Según ellos, la interacción de los siguientes factores contribuye a que un hombre maltrate a su compañera: el rechazo y el maltrato del padre, el apego inseguro a la madre y la influencia de la cultura machista, (p.117)

Es necesario recalcar que la teoría generacional de Dutton y Golant, (2012, citados por AlencarRodrigues y Cantera 2012), plantea además que, dentro de la situación de violencia de género, existe una relación entre el niño que ha presenciado la situación de maltrato de su padre hacia su madre, lo cual hace que se fortalezca una personalidad violenta, mediante un proceso de individuación. Igualmente, la separación de un niño de su madre afecta la socialización de la persona en su adultez, porque ese niño herido que tuvo que enfrentar la vida solo, percibe que puede actuar sin ayuda siempre y eso le alimenta la ansiedad por separación.

Alencar-Rodrigues y Cantera (2012) expresan al respecto que:

La capacidad que tiene el niño de tolerar la separación de su madre está condicionada por la representación interna que haya desarrollado de ella. En cambio, la incapacidad de consolarse aumenta la tensión y la ansiedad en las personas que no establecieron la constancia del objeto". (p.118).

En este sentido, existe una falla en esta etapa de desarrollo en el hombre, causada por la separación/individuación y por el hecho de que la madre no atendiera las necesidades que demandaba cuando niño, por esto, la teoría generacional de Dutton y Colant (1997), plantea que

Los hombres maltratadores muestran más probabilidades de depender de una relación y de sentir ansiedad ante la separación y la cercanía. Por lo que es probable que busquen parejas sobre las cuales puedan ejercer un control a través del cual manejen la experiencia infantil de un acercamiento fallido. (Alencar-Rodrigues y Cantera, 2012).

Desde este punto, se reconoce que la ira germina del hecho de que las necesidades de apego no son atendidas siendo un medio por el cual el niño busca el contacto apaciguador dado que, los problemas en dicha etapa originan la ansiedad a la hora de manejar la intimidad en las relaciones de pareja lo que origina que el adulto que ha padecido el abandono utilice conductas de control para reducir esta tensión ante la sensación de abandono.

En todo esto, se evidencia el gran aporte de la teoría Ceneracional de Dutton y Golant (1997. citados por Alencar-Rodrigues y Cantera, 2012) mediante la cual los autores explican que los factores psicológicos heredados a partir de una etapa anterior del desarrollo influyen en la constitución de una futura conducta violenta. De esta manera, los autores ponen de manifiesto la explicación de la violencia basada sólo en la socialización, pues argumentan que muchos hombres que han sido socializados en la misma cultura no utilizan la violencia y, por ello, proponen que la cultura refuerza la conducta una vez se ha constituido la personalidad.

Según la teoría de Cornelius Castoriadis (citado por Cancino Pérez, 2011, p.7), se definen los imaginarios sociales como

Prácticas sedimentadas de los movimientos sociales, a las instituciones que ellos mismos se dan y que varían de movimiento en movimiento y dentro de un mismo movimiento en sus distintas etapas; nos permite ir observando su diversidad y los cambios que experimentan.

ACO.USB./Vol. 20 No. 2 /PP $102-117$ /julio-diciembre-2020/Medellín, Colombia/ISSN: 16578031 E-ISSN: 2665-3354 
Aunado a esto, los imaginarios sociales son compartidos al interior de un grupo social, en el cual se elaboran esquemas cognitivos que son construidos de manera individual a través de la experiencia o de las vivencias en el grupo social al que se pertenece.

Por otro lado, los imaginarios sociales constituyen una construcción socio histórica que realizan las personas mediante la cual integran una serie de normas, símbolos, signos, instituciones y el grupo social al cual pertenece y que lo identifica. Expresa Miranda Ospino (2014, p.7), que "un imaginario no es una ficción ni una falsedad, sino que se trata de una realidad que tiene consecuencias prácticas para la vida cotidiana de las personas". Es decir el imaginario social que plantea el autor se basa en el hecho de que las ideas en las personas no son otra cosa que el reflejo de las relaciones que se producen en la sociedad y que esas ideas son autónomas y su intención se centra en modificar la realidad.

En este sentido, los imaginarios representan los mapas cognitivos en un territorio común, donde Castoriadis menciona la diferencia que existe entre una ciudad real y la ciudad imaginada por el colectivo, donde lo que es observable por el ser humano a simple vista, tal cual como se mira, desarrolla en el mismo ser humano una percepción que se desarrolla desde lo vivido o experimentado.

Desde este punto, los imaginarios sociales no son imágenes en los seres humanos, sino son el resultado de las experiencias históricas, sociales y psíquicas del sujeto, y desde ese punto los imaginarios dependen del continuo experimentar con la sociedad, con sus normas, con sus reglas, con sus condiciones y con los ajustes sociales que elabore el colectivo, desde las figuras, formas e imágenes que conllevan a una idea personal sobre ello.

En este aspecto, Cegarra, menciona que los imaginarios son significaciones sociales e institucionales que son percibidas desde un presente inmediato, las cuales estan instituidas socialmente. Castoriadis expone,

Lo imaginario del que hablo no es imagen de. Es creación incesante y especialmente indeterminada (histórico-social y psíquica) de figuras / formas / imágenes, a partir de las cuales solamente puede tratarse de 'alguna cosa'. Lo que llamamos 'realidad'y 'racionalidad', (Cegarra, 2012, p.10).

Según Murcia, Jaimes \& Gómez, los imaginarios que plantea Castoriadis se basan en la necesidad humana de establecer relaciones sociales desde sus experiencias personales, como una suma de imaginarios individuales que contienen origenes históricos, donde la significación de dichas ideas son universales y expresan una concepción personal de cada integrante de la sociedad, por esto, el autor plantea que los imaginarios no se construyen solos, emergen como creaciones del contexto social e histórico de los individuos, se integra por significaciones imaginarias sociales que a su vez, explican las situaciones que acontecen en el entorno (Murcia, Jaimes \& Gómez, 2016, pp.257-274).

En consecuencia, los imaginarios sociales son construidos por la sociedad para darle significado a los sucesos individuales de cada uno de sus miembros, es posible entonces pensar que cada sociedad tiene su propio imaginario social, y que es construido desde estas vivencias personales. Al respecto, Miranda Ospino (2014) menciona que:

La Imaginación radical: "es la capacidad de la psique de crear un flujo constante de representaciones, afectos, deseos y situaciones (rads). Es radical, en tanto es fuente de creación (poiesis del griego $\boldsymbol{\pi o t} \boldsymbol{\varepsilon} \boldsymbol{\omega}$ de hacer, crear, poner, provocar, proponer, propiciar, etcétera, según Platón es pasar del no ser al ser). Es la característica central de la psique: lo que es, es producido por la imaginación radical.

Dentro del imaginario social, la creación de una idea en particular constituye la representación personal de esa realidad para el sujeto, la cual es compartida a los demás miembros de su familia, su comunidad y su sociedad, tanto a nivel social como institucional, y lleva en su interior emociones, 
afectos, deseos, de acuerdo a lo percibido por el individuo, es por esto que para unas sociedades unos imaginarios pueden ser percibidos como positivos y se normalizan dentro de ellas y para otras este mismo imaginario es concebido negativamente y en su accionar es rechazado. Chavarro (2019) plantea que el imaginario social es un magma de significaciones imaginarias sociales, y estas son aceptadas por el colectivo social e institucional, pensar en o imaginario entiende el medio social e institucional desde el significado social que tiene para el individuo y la representación mental de la realidad, estructura la base social de las creencias sociales y sería la base de la estructura social.

En torno a los imaginarios sociales de la violencia de género, Acosta Contreras (2017, p.24), manifiesta que "se construyen de manera colectiva y se sostienen durante mucho tiempo por lo sólidos que se vuelven al transmitirse culturalmente de generación en generación.", en este caso, los imaginarios de la violencia de género son el resultado de un componente sistema de arraigos culturales que determinan los roles de género en la sociedad y que se relacionan con visiones sexistas que se fundamentan en una dinamica de poder, que a la vez marca las desigualdades y que deriva en conductas agresivas.

Una manera de comprender los imaginarios en la violencia de género, se fundamenta en el tipo de relaciones que se gesta en las familias donde se presentan agresiones e irrespeto hacia los derechos de sus miembros, lo que se evidencia a través de la violencia conyugal y el maltrato a los niños y niñas. En estos comportamientos se reproduce un ejercicio en las relaciones de poder que son direccionadas por los imaginarios individuales de dominancia del agresor, se reconocen entonces, en estas familias, formas sutiles de violencia doméstica que son trasmitidas por los padres a sus hijos en la crianza.

En estas situaciones, el agresor hace sentir a la víctima un sentimiento de culpa por su conducta de maltrato, y la víctima justifica el maltrato físico, lo cual conlleva a un maltrato psicológico que hace que la persona agredida ingrese a un círculo de maltrato del cual pocas veces sale, de ahí en muchos casos nace una violencia sexual y en los peores casos el incesto que es aceptada en la mujer por el vínculo matrimonial y la dependencia afectiva como una forma de que el hombre ejerza en poder en la familia. Una familia que es concebida como una institución social que depende de una figura masculina a nivel económico, personal y social. (Consejería Presidencial para la Equidad de la Mujer, 2004).

\section{Metodología}

La investigación se aborda desde el modelo epistémico de la fenomenología por cuanto se presenta una descripción de los imaginarios sociales de la violencia de género en el contexto de estudio. El tipo de investigación es descriptiva, con un diseño de campo transeccional por cuanto los datos se recolectan directamente desde las vivencias de la población estudiada y en un solo momento. La población de estudio la conforman 112 habitantes de la vereda Belén del municipio de Isnos (Huila), tanto hombres como mujeres que residen la zona. Para la muestra se utiliza un muestreo de tipo intencional no probabilístico, la muestra de la investigación la conformaron 18 personas con rangos de edad comprendido entre 16 y 30 años de cualquier género.

Para la recolección de los datos se utiliza la técnica de la tarea asignada, definida como una técnica que permite observar comportamientos con relación al fenómeno estudiado en participantes que son las unidades de estudio y no informantes. En el análisis de los datos se utilizan las técnicas cualitativas, las cuales, según Rodríguez Gómez, Gil Flores \& García Jiménez, (1996), "Estudian la realidad en su contexto natural, tal y como sucede, intentando sacar sentido de, o interpretar los fenómenos de acuerdo con los significados que tienen para las personas implicadas" (p.126). 


\section{Resultados de la investigación}

\section{Categoría. Elementos del lenguaje y prácticas cotidianas utilizados de manera violenta por parte de la población incluida.}

\section{Subcategoría. Lenguajes sexistas}

Se logra visualizar que en los habitantes de la vereda Belén del municipio de Isnos (Huila), existen imaginarios sociales sexistas que se relacionan con los lenguajes expresivos que utilizan elementos machistas, mediante el cual se perciben dinámicas relacionales de poder y sugestión del género masculino hacia el género femenino y de las mismas mujeres entre sí, según esto, se resalta el papel de la familia al referirse sobre la perpetuación en generaciones familiares de la violencia de género, es decir, la repetición de actos violentos por las generaciones siguientes: así, los hijos de matrimonios en que se da la violencia tienen mayor probabilidad de repetir las conductas, no solo por observarla en sus padres, sino también en aquellos casos en que son víctimas de maltrato infantil. Se interioriza entonces la violencia como una forma de relacionarse", (Cruz, Ballén, Medina, \& Rodríguez, 2014, pp.1-13).

Igualmente, se visualiza imaginarios sociales representados en los lenguajes discriminatorios, donde se coloca a la mujer en un lugar de desventaja hacia la labor del hombre. En este caso, se observa que en la vereda las mujeres son reducidas a víctimas pasivas, bajo el dominio masculino. El rol de las mujeres se limita a un papel formador de los hijos o a su rol reproductivo. Al respecto, Castoriadis (citado en Acosta Contreras, 2017) plantea que,

El imaginario radical, es una alteridad y una originación perpetua de alteridad, que figura y se figura, y al figurar esa alteridad y figurándosela, a modo de creación de imágenes que son lo que son y tal como son como figuraciones o presentificaciones de significaciones o de sentido.

En este aspecto, los imaginarios del colectivo social están sustentados en el rol dominante del hombre sobre el rol recesivo de la mujer; en este caso, existen imaginarios que se basan en experiencias previas de los individuos, y que a la vez son socialmente juzgadas por el colectivo social. Esto hace que se construya un imaginario colectivo que generaliza una condición o una situación, en este sentido las frases encasillan a las mujeres desde una postura machista y segregadora. Cruz, Ballén, Medina, \& Rodríguez, (2014, p.32), mencionan que este tipo de imaginarios son psicológicos, "refiere toda expresión verbal o no verbal dirigida hacia la pareja en forma reiterada y que causa daños emocionales por el contenido de sentimientos negativos hacia la persona afectada y que se constituye como léxico popular".

\section{Subcategoría. Prácticas cotidianas}

Dentro de las prácticas cotidianas de los participantes de la muestra, se evidencia que existen imaginarios sociales en torno a los roles que ejercen cada uno de los habitantes en el hogar, en este caso, se identifican imaginarios de violencia conyugal que denotan una incidencia en la familia de la construcción de imaginarios en las prácticas cotidianas, que denotan a la mujer como la responsable de las labores del hogar y al hombre como proveedor al hogar, lo cual le da un derecho de socialización que es negado a la mujer.

Estos imaginarios son concebidos intergeneracionalmente a través de modelos parentales, desde la teoría generacional que plantean Alencar-Rodrigues y Cantera, (2012), se demuestra que: 
Los hombres maltratadores muestran más probabilidades de depender de una relación y de sentir ansiedad ante la separación y la cercanía, por lo que es probable que busquen parejas sobre las cuales puedan ejercer un control a través del cual manejen la experiencia infantil de un acercamiento fallido (p.119).

Estos esquemas de conducta tienen su trasfondo de las vivencias de la niñez y evidencian que existe una relación entre los procesos educativos y los sucesos generadores de violencia.

\section{Subcategoría. Roles de Género}

Ante los roles de género, se evidencia que los imaginarios se centran en las labores hogareñas en las cuales el principal papel lo tiene la mujer, además se denota que en los hombres existe aún la percepción de que la mujer tiene que presentar conductas servilistas, ante lo cual se identifica un tipo de "violencia indirecta", dentro de la cual el hombre es quien económicamente provee a los gastos del hogar, y la mujer se niega a contribuir al sostenimiento del hogar. En los imaginarios se evidencia que el hombre le prohíbe a la mujer trabajar o estudiar, en este caso, también se denota que en los roles asumidos por el hombre relega la mujer para tener contactos sociales y controla por ende sus actividades y amistades, todo esto enmarcado en un contexto de limitación de la libertad de las mujeres y su posibilidad de tomar decisiones de acuerdo con sus propios criterios y deseos.

Al tiempo, Alencar-Rodrigues y Cantera (2012), expresan que, "la influencia del maltrato y de la disfunción familiar en la niñez a través de experiencias que afectan al sentido de identidad hace que el niño recurra con más probabilidad a la cultura para justificar su violencia ", (p.24), lo cual ocasiona un impacto negativo en el papel de la mujer en el entorno social de la vereda. Además, se denota un imaginario social enfocado hacia el control del comportamiento social por parte de la Iglesia como Institución, es decir, se percibe cierta represión por parte de la simbología que la religión representa. Al respecto, Castoriadis, (1996, citado por Miranda Ospino, 2014), expresa que ante cualquier riesgo de cambio se impone por la fuerza, en ejercicio de la coerción y la coacción mediante la aplicación de sanciones y castigos.

En este sentido, ante la pasividad del individuo y el colectivo la institución imaginaria de la sociedad establecida, asegura su permanencia, expresa Castoriadis (1996, citado por Miranda Ospino, 2014, p. 45), "mediante la formación (elaboración) de la materia prima humana en individuo social, en el cual se incorporan tanto las instituciones mismas como los "mecanismos" de la perpetuación de tales instituciones”. (p.45). De esta manera, el imaginario está supeditado al ejercicio de poder de la iglesia en los habitantes, la cual manipula las formas de vestir de las mujeres, lo cual las excluye de su propio deseo y las mantiene en un encasillamiento social que la relega a liberar su forma de vestir.

Al respecto Castoriadis (1996, citado por Miranda Ospino, 2014) indica que

Las significaciones imaginarias sociales y las instituciones correspondientes se convertirán tendencialmente en un Leviatán, ese monstruo bíblico que menosprecia toda cosa alta y de esta forma impone un esquema de las cosas hacia la destrucción, e inicialmente transforma al individuo en un ciudadano servil, apático, temeroso, sin capacidad de cuestionar, porque se imita a reproducir los patrones aprendidos en su proceso de socialización por la institución instituida (p.86).

Estos imaginarios de roles de género dan lugar a etiquetas y señalamiento dentro de las sociedades que imponen creencias machistas que afectan la vida cotidiana, y que son aceptadas por las personas como parte de una cultura, pero que colocan a la mujer en una postura de servicio y responsabilidad en el hogar a la vez que direccionan su forma de actuar desde unos parámetros socialmente establecidos, con una reafirmación de superioridad del hombre, lo cual hace que aparezca un tipo de imaginario radical que se instaura en las familias y en las instituciones sociales, dando lugar a la justificación de la violencia de género. 


\section{Subcategoría. Pensamientos frente al género}

Dentro de los pensamientos frente al género se evidencia en los habitantes de la vereda participantes en el estudio que no existe una definición clara y precisa de las tipologías de la violencia de género y los conceptos incluidos en esta definición. En algunos casos son confundidos con machismo, posiblemente sea por el contexto rural y a la doblegación de la culpa y la represión de la mujer, en este caso, existe una serie de imaginarios radicales que se relacionan con conductas estigmatizadoras y excluyentes, las cuales hacen parte de un aprendizaje generacional que ha sido reforzado socialmente, en este caso, los manierismos de un hombre son mal vistos tanto por hombres como por las mujeres y constituyen un Imaginario Radical. Al respecto expone Acosta Contreras (2017),

Es la capacidad de la psique de crear un flujo constante de representaciones, afectos, deseos y situaciones radicales, en tanto es fuente de creación (poiesis del griego $\boldsymbol{\pi} \mathbf{0} \mathbf{\varepsilon} \boldsymbol{\omega} \boldsymbol{\omega}$ de hacer, crear, poner, provocar, proponer, propiciar, etcétera, según Platón es pasar del no ser al ser). Es la característica central de la psique: lo que es producido por la imaginación radical (p.12).

Además, se encontraron vacíos conceptuales y en las formas de manifestación acerca de lo conceptos machismo, hembrismo y feminismo.

\section{Subcategorías. Conductas discriminatorias de género}

Frente a las conductas discriminatorias se evidencia que en la población de la muestra aún se presentan conductas de discriminación por parte de los hombres hacia las mujeres, orientadas por una carga negativa que se da acerca de la mujer y la feminidad por parte del hombre, como también, una subvaloración cultural de la mujer por parte del hombre. Esto da lugar a una discriminación en la labor de la mujer minimizando su importancia, además se visualizó que las mujeres atacan, critican y excluyen a sus congéneres que se visten o desempeñan un papel diferente a los roles establecidos socialmente en la comunidad, lo que se percibe como una postura machista.

\section{Categoría. Esquemas de violencias de género presentes en los imaginarios sociales identificados}

\section{Subcategoría. Violencia Física}

Se evidencia que la violencia física dentro de los imaginarios identificados es socialmente aceptada, y se denota que las mismas mujeres justifican estos tipos de violencia, se enmarcan además congruencias en los simbolismos relacionados con la violencia de género, lo cual hace que se justifique la relación de desigualdad de poder en la pareja. Se infiere que los sujetos de investigación consideran la violencia de género como algo problemático, de connotación negativa, que afecta la integridad personal y familiar. En los resultados se identifican imaginarios sociales que incluyen motivos para permitir ejercicio de la violencia con las mujeres, tales como la dependencia económica y afectiva con el agresor o problemas interpersonales como la falta de autoestima.

\section{Violencia Psicológica}

En torno a las violencias psicológicas, se evidencia que en la vereda aún se siguen presentando construcciones y esquemas mentales orientados a la sujeción de la mujer desde el poder y la dominancia del hombre, como también se resalta el papel del hombre sobre el de la mujer, producto de los ingresos económicos que provee al interior del hogary la provision que le brinda a los miembros de la familia. Igualmente, se evidencia chantaje emocional en cada uno de los imaginarios que limita la acción de la mujer, con una demarcación de superioridad del hombre sobre la mujer. 


\section{Violencia Emocional}

En torno a la violencia emocional dentro de los imaginarios también se percibe en las mujeres cierta normalización a condiciones de violencia psicológica enmarcadas en denigración de su emocionalidad, de los gritos, insultos o malas palabras, o de las burlas por el aspecto físico o las capacidades intelectuales de la mujer, se denota que aún persiste la superioridad del hombre sobre la mujer.

\section{Violencia Sexual}

Por otro lado, en cuanto a la violencia sexual se presentan estigmas y discriminaciones a las mujeres que no se encasillan al papel tradicional de la mujer. Entre las mujeres se presentan conductas machistas reflejadas en críticas y exclusión de otras mujeres por su aspecto físico, formas de vestir o actuar.

\section{Privación Económica y Patrimonial}

En torno a lo económico se observó que en la vereda existe cierta dependencia económica de la mujer hacia el hombre, en este caso, el hombre persiste en sus conductas de machismo, con acciones de relego hacia las capacidades de la mujer y castrando cada una de sus participaciones en el contexto laboral.

\section{Violencia Simbólica}

En cuanto a la violencia simbólica, se denota en la población que aún persiste la dominancia del hombre sobre la participación de la mujer, en este caso, se evidencia en los habitantes patrones estereotipados, mediante mensajes denigrantes en contra de las mujeres, los cuales son transmitidos entre padres e hijos. Estas conductas hacen que se reproduzca la dominación, desigualdad y conductas de discriminación en las relaciones sociales de la comunidad, lo cual naturaliza la subordinación de la mujer en la sociedad.

\section{Categoría. Vivencias individuales a través de la aplicación de entrevistas a la población en torno a estos imaginarios}

\section{Subcategoría. Vivencias descritas sobre violencia de género en la vereda}

Se evidencia que en la vereda se presentan conductas de violencia enmascaradas y que no son socializadas abiertamente por sus pobladores, las vivencias individuales de la población se retoman desde conductas de poder y dominancia del rol masculino sobre el género femenino, los casos de violencia se presentan reiteradamente bajo patrones comportamentales repetidos intergeneracionalmente, en este sentido los esquemas de violencia y las vivencias de la comunidad son machismo tanto por los hombres como las mujeres.

La mayor representación de la violencia de género en la comunidad son los golpes, los cuales son normalizados por sus habitantes. 


\section{Categoría. Imaginario social frente a los casos de violencias de género presentados en la vereda}

\section{Subcategoría. Imaginarios sociales}

En los resultados obtenidos de los talleres realizados se evidencia que la población presenta una serie de imaginarios sociales comunes que han sido construidos en torno a las conductas machistas y discriminatorias, que se retoman intergeneracionalmente y son reforzadas mediante imaginarios sociales productos de vivencias personales. Los imaginarios de violencia de género identificados en la comunidad giran en torno a la incidencia de conductas machistas formadas por la familia, durante la niñez. En este sentido, se pone de manifiesto el impacto de las vivencias familiares de las personas en sus futuras conductas violentas.

Los imaginarios sociales se construyen en este caso de forma individual a partir de las experiencias obtenidas en la vereda, los cuales fueron poco socializados, pero dejaron ver imaginarios discriminatorios, segregadores y de violencia, que en la mayoría de las veces se orientaban a posturas machistas de los hombres y de las mujeres hacia ellas mismas. Se evidencia la influencia social en la construcción de roles favorecedores de la agresión y la repetición del ciclo de violencia de género, tal como lo mencionan los sujetos de investigación.

\section{Conclusiones}

A partir del proceso desarrollado se puede concluir que en la población de la vereda Belén del municipio de Isnos, hombres y mujeres entre los 16 y 30 años existe la presencia de imaginarios sociales discriminatorios que afectan las dinámicas relacionales entre las mujeres y los hombres, con un marcado papel de dominancia del hombre y la sujeción por parte de la mujer. Igualmente, persiste el papel discriminadory de segregación hacia la mujer.

Con relación a las concepciones de violencia de género se observó que no existen conceptos claros en la unidad de estudio. Existe una normalización de las conductas de violencia tanto física, psicológica como emocional en la población, las cuales son trasmitidas intergeneracionalmente de padres a hijos en el papel formador de la familia y la iglesia. El imaginario en este sentido, en esta comunidad existe un ejercicio de poder de la iglesia, quien limita la actuación de la mujer en su acción personal de presentación y actuación.

Por último, se denota que desde el rol de género, cada participación de la mujer en los contextos sociales está delimitada hacia labores hogareñas y la crianza de los hijos, lo cual anula la participación de la mujer en la socialización dentro de la comunidad. Esto limita su su proyecto de vida en lo referente a la profesionalización.

\section{Recomendaciones}

Es importante generar espacios de reflexión comunitaria que permitan desde los mismos actores sociales desarrollar procesos encaminados a que las mujeres comprendan que en efecto se requiere un proceso de igualdad entre los roles asumidos por los hombres y las mujeres para garantizar un trato igualitario entre los dos y permitir que se minimicen este tipo de imaginarios que discriminan a las mujeres y las segregan descalificando su participación en la comunidad.

Por medio de los resultados de la Investigación se deben desarrollar articulaciones interinstitucionales con las Juntas de Acción comunal de la vereda, sus habitantes, las entidades de salud como la ESE Municipal San José, las entidades territoriales como la Alcaldía Municipal, la oficina de la mujer, la Comisaria de familia del municipio y la Personería municipal, para desarrollar talleres preventivos sobre la violencia de género, el machismo, y la misoginia en la comunidad. 
Se deben desarrollar procesos de sensibilización con la comunidad donde se le brinde las herramientas informativas acerca de la Ruta Integral de Atenciones en caso de Violencia de Cénero y las entidades responsables de su manejo.

Se recomienda realizar por medio del ente territorial procesos de articulación con grupos feministas que realicen ponencias, talleres participativos, y sensibilizaciones con la comunidad acerca de la importancia de establecer procesos de prevención de la violencia de género en el municipio, empoderando a los miembros de la comunidad como líderes participativos.

Se recomienda desmitificar los imaginarios construidos por posturas machistas en las mujeres de la vereda y fortalecer acciones desde la igualdad que permitan la participación de las mismas en la sociedad, mediante un trato justo e igualitario, siendo visibilizadas, desestructurando las percepciones sociales construidas.

Se recomienda desde la práctica académica del psicólogo en formación de la Corporación Universitaria Minuto de Dios, desarrollar más procesos de investigación en las comunidades con este tipo de temáticas, logrando ampliar la mirada en torno a los imaginarios sociales de la violencia de género, al tiempo, desarrollar procesos de capacitación a la comunidad sobre los mecanismos de acción ante estos casos, permitiendo brindar a la comunidad afectada la información necesaria para prevenir y mitigar el impacto de la violencia de género en el individuo.

\section{Referencias bibliográficas}

Acosta Contreras, F. (2017). Imaginarios sociales en la violencia de género. Enfoque caribe, 1-28.

Águila Gutiérrez, Y.; Hernández Reyes, V.; \& Hernández Castro, V. (2016). Las consecuencias de la violencia de género para la salud y formación de los adolescentes. Revista Médica Electrónica, 38(5), 697-710. Recuperado en 02 de octubre de 2020, de http://scielo.sld.cu/scielo.php?script=sci_arttext\&pi$d=$ S1684-18242016000500005\&lng=es\&tlng=es

Alencar-Rodrigues, R.., \& Cantera, L. (2012). Violencia de Género en la Pareja: Una Revisión Teórica. PSICO, 44, 116-126.

Cancino Pérez, Leonardo. (2011). Aportes de la noción de imaginario social para el estudio de los movimientos sociales. Polis (Santiago), 10(28), 69-83. https://dx.doi.org/10.4067/S071865682011000100005

Cegarra, J. (2012). Fundamentos Teórico Epistemológicos de los Imaginarios Sociales. Cinta de Moebio, 43, $1-13$.

Chavarro, L. (2019). Lo imaginario social en el mundo digital. Apuntes sobre dos investigaciones acerca de las Nuevas Tecnologías e Internet. PLURIVERSIDAD, 3(3), 141-168. https://doi.org/10.31381/pluriversidad.v3i3.2240

Comisaria de familia de Isnos (Huila). (2018). Casos de Violencia de Cénero. Isnos, Huila: Alcaldia Municipal Isnos Huila.

Consejería Presidencial para la Equidad de la Mujer. (2004). La familia: Reflexiones, cifras y legislación sobre violencia intrafamiliar. Observatorio de asuntos de género, 1-20.

Cruz, T., Ballén, P., Medina, S., \& Rodríguez, A. (2014). Imaginarios sociales sobre la violencia conyugal. Revista Senderos Pedagógicos, 5, 37-49.

Fernández Moreno, S. Y. (2017). Salud y violencia de género en América Latina: Una lectura género sensible y sociosanitaria. Antioquia, Colombia: Red Colombiana de Mujeres por los Derechos Sexuales y Reproductivos RCMDSR.

Hernández Sampieri, R., Fernández Collado, C., \& Baptista Lucio, M. d. (2016). Metodología de la Investigación. México: Mc Graw Hill. 
Instituto Nacional de Salud. (2018). Violencias de género e intrafamiliar. Comportamiento de la vigilancia en salud pública Colombia 2018. Boletin Epidemiológico Semanal, 21, 1-32. https://www.ins.gov.co/ buscador-eventos/BoletinEpidemiologico/2018\%20Bolet\%C3\%ADn\%2oepidemiol\%C3\%B3gico\%20semana\%2021.pdf

Miranda Ospino, E. A. (2014). El imaginario social bajo la perspectiva de Cornelius Castoriadisy su proyeccion en las representaciones culturales de Cartagena de Indias. Cartagena de Indias: Universidad de Cartagena.

Murcia, N.; Jaimes, S.; \& Cómez, ]. (2016). La práctica social como expresión de humanidad. Cinta de moebio, (57), 257-274. https://dx.doi.org/10.4067/S0717-554X2016000300002

Organización Mundial de la Salud [OMS]. (2017). Comprender y abordar la violencia sexual contra las mujeres. España: OMS.

Organización Mundial de la Salud [OMS]. (2018). Hechos y cifras: Acabar con la violencia contra mujeres y niñas. Prevalencia y efectos de la violencia conyugal y de la violencia sexual no conyugal en la salud. http://www. unwomen.org/es/what-we-do/ending-violence-against-women/facts-and-figures

Riffo Pavón, I. (2016). UNA REFLEXIÓN PARA LA COMPRENSIÓN DE LOS IMAGINARIOS SOCIALES. Comuni@cción, 7(1), 63-76. Recuperado en 02 de octubre de 2020, de http://www.scielo.org.pe/scielo. php?script=sci_arttext\&pid=S2219-71682016000100006\&Ing=es\&tlng=es.

Secretaria de Salud Departamental del Huila. (2018). Boletin Epidemiológico. Neiva, Huila: Gobernación del Huila.

Segato, R. L. (2003). Las estructuras elementales de la violencia. Quito Ecuador: Derechos Humanos. Viejos problemas, nuevas miradas.

Sistema Nacional de Vigilancia en Salud Pública [SIVICILA]. (2018). Casos de violencia de género en Colombia. Bogotá, D.C: Ministerio de Salud-Colombia.

Rodríguez Cómez, G.; Gil Flores, J.; García Jiménez, E. (1996). Metodología de la investigación cualitativa. Granada (España): Ediciones Aljibe 